\title{
Female paraurethral leiomyoma: a case report
}

\author{
Farhat Mazhari ${ }^{*}$, Roopam Singh ${ }^{1}$, Kirti Anima Kerketta' ${ }^{1}$ Nilay Pathak ${ }^{2}$ and Neha Singh ${ }^{1}$
}

\begin{abstract}
Background: Leiomyoma is a benign fibromuscular neoplasm originating from smooth muscle cells. Paraurethral leiomyoma is an extremely rare, benign, hormone-dependent growth from the mesenchymal cells in paraurethral space of female urethra. They usually appear in the reproductive age group women, mean age of presentation being approximately 41 years.

Case presentation: A 48-year-old woman presented with palpable mass at vagina and weak urinary stream. She underwent clinical examination and CT scan and MRI imaging followed by transvaginal excision of the mass. Histopathological examination confirmed paraurethral leiomyoma.

Conclusions: Paraurethral leiomyoma owing to its rare incidence poses a diagnostic dilemma for the gynecologists. Radiological imaging techniques help in diagnosis. Surgical excision via vaginal route is the treatment of choice. Diagnosis is confirmed by histopathology.
\end{abstract}

Keywords: Benign, Fibroid, MRI, Paraurethral leiomyoma, Smooth muscle

\section{Background}

Leiomyomas are rare benign fibromuscular tumors developing from smooth muscle cells. Most frequently they occur in the gastrointestinal and genitourinary system. Paraurethral leiomyoma has exceptionally rare incidence [1]. It consists of $5 \%$ of all paraurethral masses occurring in 1:1000 women [1]. Only few cases of true paraurethral tumors have been reported among the reported cases of urethral tumors. It grows as a mesenchymal neoplasm from the paraurethral space or vesicovaginal septum without any communication with urethra, bladder or vagina [1]. Patients present with wide variety of symptomatology varying from asymptomatic to urinary retention and voiding dysfunctions. Surgical excision is needed for symptomatic cases.

We report a case of a true paraurethral leiomyoma confirmed by histopathology in a young woman.

\footnotetext{
*Correspondence: farhatmazhari1987@gmail.com

${ }^{1}$ Department of Obstetrics and Gynecology, Bokaro General Hospital, Bokaro Steel City, Jharkhand 827004, India

Full list of author information is available at the end of the article
}

\section{Case presentation}

A 48-year-old, P4L3 woman attended Gynae OPD with chief complaints of a mass protruding at the vaginal introitus with incomplete voiding of urine and weak urinary stream for past two years. There was no associated menstrual complaint or abdominal pain or bleeding from mass. She did not complaint of dysuria, dyspareunia. Past medical and surgical history was insignificant. On physical examination, the abdomen was soft, nontender and no organomegaly or lump was elicited. On local examination of the perineum, a $7 \times 5 \times 4 \mathrm{~cm}$ firm, smooth, non-tender, non-fluctuant mass was appreciated at the anterior wall of vagina posterior to urethral meatus (Fig. 1). An ulcer of area around $2 \times 2 \mathrm{~cm}$ was seen in the central part of the mass. Per vaginal examination revealed anteverted, parous uterus and normal adnexa.

A pelvic ultrasonography (USG) was done which showed a bulky uterus with features of chronic cervicitis and a small hemorrhagic cyst in left ovary. Abdominopelvic computed tomography (CT) scan imaging identified an inhomogeneous mass from urethra or periurethral region. Pelvic magnetic resonance 


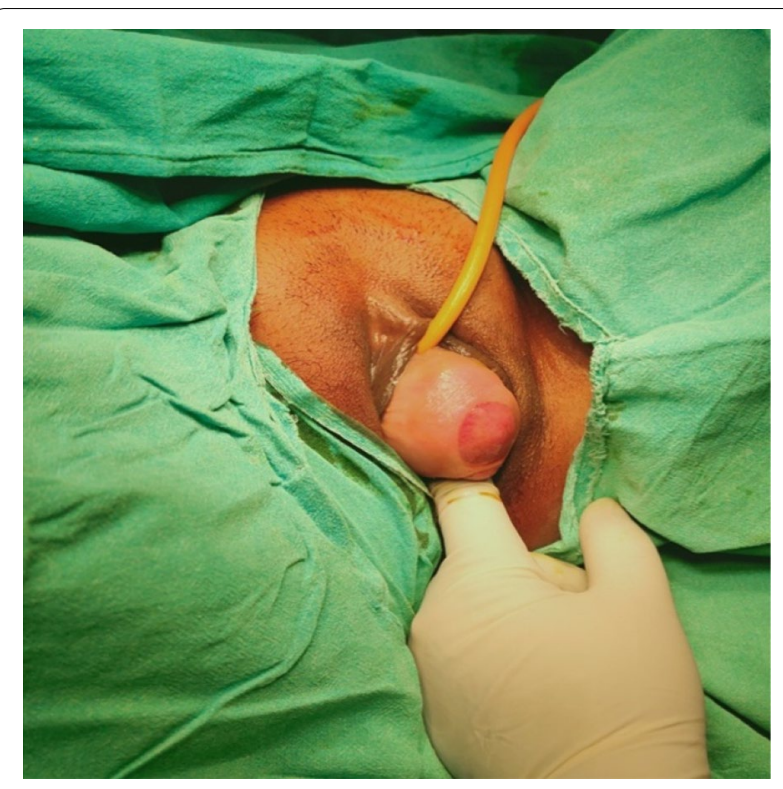

Fig. 1 Paraurethral leiomyoma in situ

imaging (MRI) was done where T1-weighted images showed well-defined encapsulated enhancing solid perineal mass in the periurethral region suggestive of urethral/ paraurethral leiomyoma (Fig. 2a, b). There was no urethral invasion. A fine needle aspiration cytology (FNAC) from the mass through transvaginal route revealed spindle cell lesion suggestive of leiomyoma. Preoperative cystoscopy and urethroscopy showed no involvement of bladder and urethra.

Decision for surgery was taken with consultation of urologists and surgeon team. After urethral catheterization with 16-French Foley catheter, hydro dissection was done with injection of normal saline over the vaginal submucosa. Sub-urethral vertical incision was given over the mass and enucleation was done. Dead space was obliterated with Vicryl 2-0 sutures, redundant portion of vaginal mucosa excised and colporrhaphy was done. The excised specimen was encapsulated tumor, $6 \mathrm{~cm}$ (length) $\times 4 \mathrm{~cm}$ (width) $\times 4 \mathrm{~cm}$ (height) in size and cut section showed firm, whitish whorled appearance. Histopathology report showed interlacing fascicles of smooth muscle cells with hyaline degeneration thus confirming it to be leiomyoma (Fig. 3a, b).

Foley catheter was removed after $48 \mathrm{~h}$ and patient was discharged. Postoperative clinical course was uncomplicated. At four-week follow-up visit, patient was examined. The surgery site was clean, healing well and she was relieved of her symptoms.

\section{Discussion}

Paraurethral leiomyomas are benign tumors of mesenchymal origin [2]. The etiology is unknown [3] but the majority of reported cases in reproductive age women explains the hormonal role in its development [4]. Paraurethral leiomyoma affects both genders but has a female preponderance [4] and usual age of presentation is around 40 to 44 years [3]. Patients present with variable symptoms of pelvic pressure, urinary retention, protruding mass, voiding dysfunction lower urinary tract symptoms or vaginal bleeding. $[5,6]$. The differential diagnosis of mass in this area can be urethral leiomyoma, urethral prolapse, urethral caruncle, urethral diverticulum, Bartholin's duct cyst, Skene's duct abscess, Gartner's duct cyst and urethral carcinoma [1]. No malignant

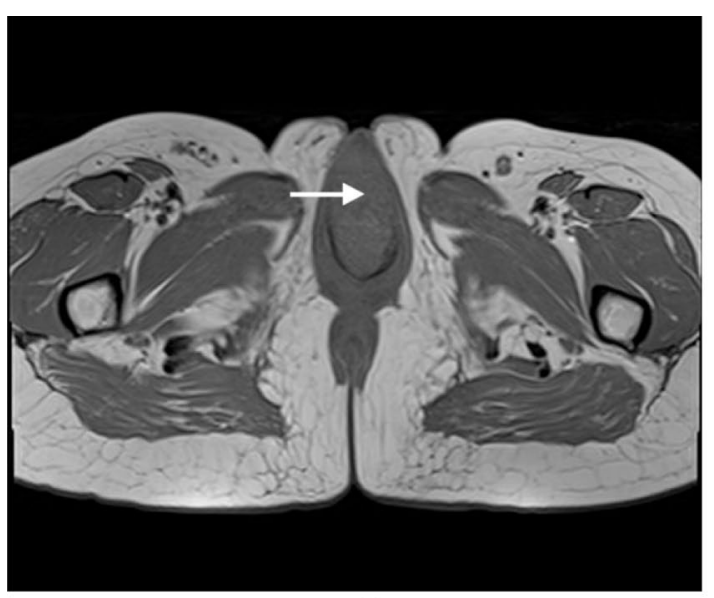

a

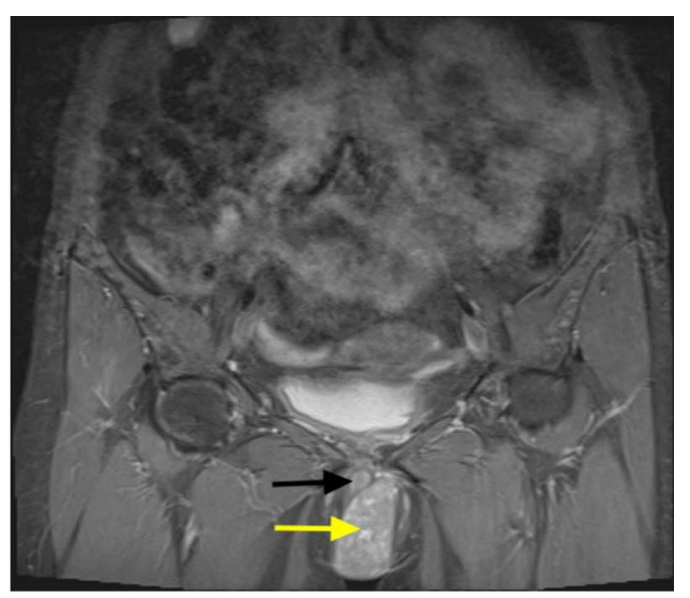

b

Fig. 2 Pelvic MRI T1-weighted images a Axial scan showing isointense mass b Coronal scan showing contrast enhancement of mass (yellow arrow), posterolateral to urethra (black arrow) 


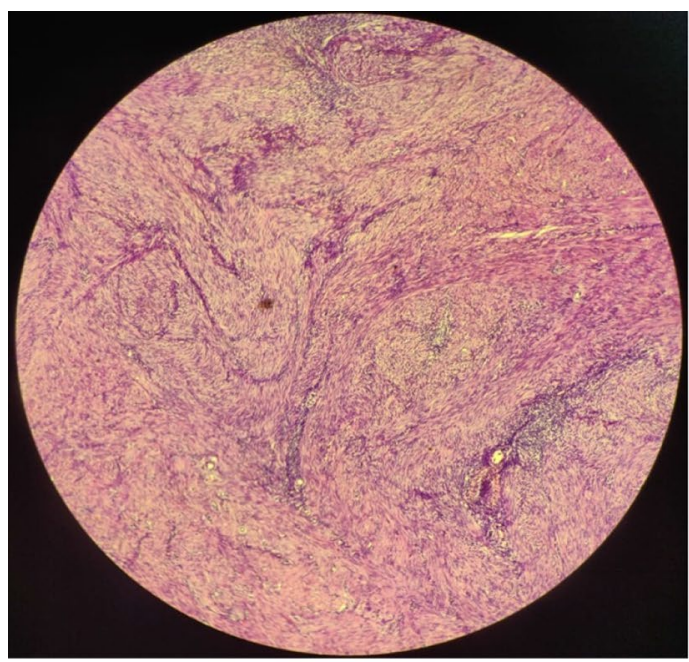

a

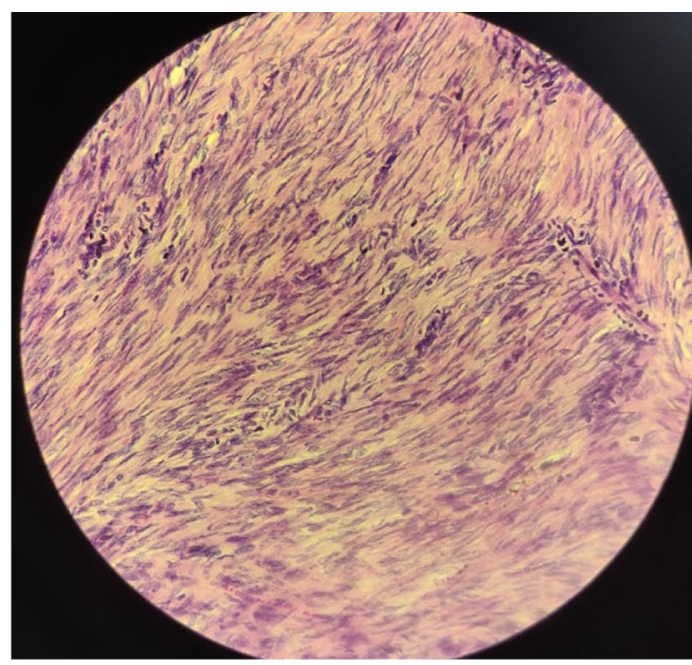

b

Fig. 3 Histopathology picture showing interlacing fascicles of spindle-shaped cells with oval nuclei and eosinophilic cytoplasm

transformation and very low risk of recurrence of this entity has been reported. To date, only four patients have developed recurrence [7]. Paraurethral leiomyomas are often mobile while urethral leiomyomas are fixed as seen in our case.

Detailed history and careful clinical examination are significant in diagnosis and evaluation of this case [6]. Radiological imaging techniques like USG, CT scan and pelvic MRI help in exact location and origin of mass and relationship with nearby structures which is essential for surgical planning. Transvaginal, transperineal, translabial USG has a role, which will show well-defined isoechoic or hypoechoic homogeneous mass and inner vascularity on color flow Doppler. MRI will show leiomyoma as homogeneous well-encapsulated mass appearing hypointense or isointense on T1-weighted images and intermediate signal on T2-weighted images [7].

Local surgical excision via transvaginal route has been recommended as the mode of management $[4,6,8]$. For larger paraurethral leiomyomas, abdominoperineal approach may be necessary. Histopathology is the gold standard for diagnosis which shows interlacing fascicles of spindle-shaped smooth muscle cells with oval nuclei and eosinophilic cytoplasm under hematoxylin and eosin stain [9]. Immunohistochemistry is positive for desmin and smooth muscle cell antigen [10].

The patient in our case presented at the age of 48 years with a palpable mass at the vaginal introitus and voiding difficulties which is similar to the symptoms reported in previous case reports [5, 6, 8]. After preoperative evaluation, the mass was surgically excised through transvaginal route. Symptoms were resolved and postoperative recovery was uneventful.

\section{Conclusion}

Paraurethral leiomyoma is a rare benign tumor of female genitourinary tract. Its anatomical relation to bladder, urethra and vagina makes it an important differential diagnosis of paraurethral masses in women of reproductive age group. Radiological imaging techniques like USG and MRI play invaluable role in its diagnosis. Diagnosis is confirmed on histopathological examination which shows spindle-shaped cells arranged in whorls or fascicles. Surgical excision via vaginal route is the treatment of choice which should be done meticulously to avoid urethral injury. However, abdominal approach may be needed in some cases.

\section{Acknowledgements \\ We would like to acknowledge the contribution of Dr. Prakash Pandey (Department of Pathology) in helping us with proper histopathological diagnosis and pictures.}

\section{Authors' contributions}

FM and RS concepted the layout of case study and drafted the article. KAK and NP operated on the patient and made substantial contribution in critical revision of the article. NS contributed to clinical follow-up and collection of radiological imaging data. All authors read and approved the final version of manuscript.

\section{Funding}

The case study did not receive any funds from any source.

\section{Availability of data and material}

Data sharing is not applicable to this article as no datasets were generated or analyzed during the current study. 


\section{Declarations}

Ethics approval and consent to participate

Ethical clearance (Certificate number- Ref/Med/2021-72) was obtained from the Ethical Committee of Bokaro General Hospital.

\section{Consent for publication}

Written informed consent was obtained from the patient for publication of this case report and accompanying images.

\section{Competing interests}

The authors have no conflicts of interests to declare.

\section{Author details}

${ }^{1}$ Department of Obstetrics and Gynecology, Bokaro General Hospital, Bokaro Steel City, Jharkhand 827004, India. ${ }^{2}$ Department of Surgery, Bokaro General Hospital, Bokaro Steel City, Jharkhand 827004, India.

Received: 15 March 2021 Accepted: 3 June 2021

Published online: 10 June 2021

\section{References}

1. Rezai S, Bue SL, Bahl N, Chadee A, Gottimukkala S, Fishman A et al (2017) A true paraurethral leiomyoma, a case report and review of literature. Obstet Gynecol Int J 6(5):00218
2. Aydogmus S, Aydogmus H, Ekmekci E, Keleckci S, Eris S (2017) Paraurethral Leiomyoma. J Reprod Med 62(1-2):85-88 (PMID: 29999298)

3. Badr MR, Ahmad Hizagy AMG, Said Abuelbaga MMR, Mahmoud Mostafa DE (2019) Paraurethral leiomyoma during pregnancy: a case report and review of literature. J Clin Trials 9:370

4. Migliari R, Buffardi A, Mosso L (2015) Female paraurethral leiomyoma: treatment and long-term follow-up. Int Urogynecol J 26(12):1821-1825

5. Chodisetti S, Namburi RR, Boddepalli Y (2015) Female urethral leiomyoma presenting with acute urinary retention: a rare case with unusual presentation. Indian J Surg 77(suppl 1):128-129

6. Fridman D, Abeshouse M, Sankin A (2018) Paraurethral leiomyoma as an incidental finding in patient with fibroid uterus. Case Rep Obstet Gynecol 2018:7042960

7. Jiménez Navarro M, Ballesta Martínez B, Rodríguez Talavera J, Amador Robayna A. Recurrence of urethral leiomyoma: a case report. Urol Case Rep. 2019:26:100968.

8. Harada J, Ishikawa Y, Fujiwara H, Ishihara G (2018) Female paraurethral leiomyoma successfully excised through a vaginal approach: a case report. J Obstet Gynaecol Res 44(6):1174-1176

9. Braga A, Soave I, Caccia G, Regusci L, Ruggeri G, Pitaku l et al (2020) What is this vaginal bulge? An atypical case of vaginal paraurethral leiomyoma. A case report and literature systematic review. J Gynecol Obstet Hum Reprod. 31:101822

10. Beng Kwang N, Naidu A, Yahaya A, Pei SL (2016) Urethral leiomyoma: a rare clinical entity. Case Rep Surg 2016:6037104

\section{Submit your manuscript to a SpringerOpen ${ }^{\circ}$ journal and benefit from:}

- Convenient online submission

- Rigorous peer review

- Open access: articles freely available online

- High visibility within the field

- Retaining the copyright to your article

Submit your next manuscript at $\boldsymbol{\nabla}$ springeropen.com 